\title{
Abundancia y distribución de Aedes aegypti (Diptera: Culicidae) y dispersión del dengue en Guasave Sinaloa, México
}

\section{Cipriano García ${ }^{1}$, Lourdes García ${ }^{1}$, Leticia Espinosa-Carreón T. ${ }^{1}$ \& César Ley P. ${ }^{2}$}

1. Laboratorio de Bioinsecticidas. Centro Interdisciplinario de Investigación para el Desarrollo Integral Regional (CIIDIR-COFAA-IPN) Unidad Sinaloa. Blvd. Juan de Dios Bátiz Paredes \#250. C.P. 81101. Guasave Sinaloa, México; cgarciag@ipn.mx,mlgv82@hotmail.com, tespinosac@ipn.mx

2. Departamento: Vectores y Zoonosis Delegación Sanitaria No. 4 de la SS (Servicios de Salud de Sinaloa). Blvd. Francisco Zarco s/n, Col. Rosales. C.P. 80230. Culiacán Sinaloa, México; leyq@gmail.com

\author{
Recibido 12-X-2010. Corregido 10-II-2011. Aceptado 17-III-2011.
}

\begin{abstract}
Abundance and distribution of Aedes aegypti (Diptera: Culicidae), and dengue dispersion in Guasave Sinaloa, México. Dengue is an important disease that affects humans, and is transmitted by A. aegypti. During 2006, a total of 477 cases of hemorrhagic dengue, and 1510 of classic dengue were recorded in Sinaloa. Due to this high impact, a study on insect abundance and distribution, as well as their relationship with dengue dispersion, was carried out from April 2008 to March 2009 in Guasave, Sinaloa. The study included a total of six sectors in the city, considering 16 colonies; besides, 96 traps were distributed in these sectors to monitor the vector population density and female number per trap. The adult density index (ADI) and traps positive index (TPI) were calculated, and data were used to obtain the monthly dengue dispersion maps. The highest abundance of female (137 and 139) was found in July and August. In August and September the sectors 2, 4 and 6 showed the highest ADI values 2.44, 3.35 and 2.290, while TPI values were 56.25, 58.82 and 61.54 , with the highest epidemiology dengue dispersion. The Pearson correlation $(\mathrm{p}<0.05)$ showed better values with the precipitation $(\mathrm{r}=0.80)$ than mean temperature $(\mathrm{r}=0.76)$ with zero lag months; while the cases of classic dengue and hemorrhagic dengue $(\mathrm{CD} / \mathrm{HD})$ is mostly related with the precipitation $(\mathrm{r}=0.98)$, and minimum temperature $(\mathrm{r}=0.79)$, with two lag months. In conclusion, the ADI and TPI index, as well as the maps obtained, allowed us to know the location of epidemiologic dengue risk areas. This information can be used to develop better control measures biological and chemical for the mosquito, in this location. Rev. Biol. Trop. 59 (4): 1609-1619. Epub 2011 December 01.
\end{abstract}

Key words: mosquito, risk, classic dengue, hemorrhagic dengue, Guasave Sinaloa México.

El dengue es una enfermedad viral en humanos, la cual es transmitida por el mosquito Aedes aegypti (L) principal vector en América. En el 2006 se registraron 27287 casos en México, de los cuales 22810 fueron de dengue clásico (DC) y 4477 de dengue hemorrágico (DH). En este año, el Estado de Sinaloa tuvo el mayor número de casos de DH a nivel Nacional (SSA 2007), mientras que en el periodo 2000-2006 tuvo también el mayor número de casos de DC. Por la importancia epidemiológica del virus y por los nuevos brotes de la enfermedad en diversas ciudades del país en las que no se tenía registro de su ocurrencia, se han realizado estudios enfocados a conocer la distribución espacio-temporal del mosquito $A$. aegypti y su relación con los factores del clima, como base para medir el riesgo de dispersión de la enfermedad.

El muestreo del insecto se realiza comúnmente con el uso de trampas para recolectar adultos y huevecillos, de esta manera el número de individuos capturados por trampa se utiliza para calcular los índices larvario en el monitoreo del vector (WHO 1995). Sin embargo, el índice no considera variables epidemiológicas 
para determinar el riesgo de transmisión, ni la abundancia del adulto como elementos para la identificación rápida de áreas con mayor riesgo del dengue (Focks 2003). Otras técnicas de muestreo poblacional permiten calcular el índice de trampas positivas (ITP): se considera como trampa positiva aquella que capturó por lo menos a una hembra (Carbajo et al. 2006), y el índice de densidad de adultos (IDA), que permite conocer además la fluctuación poblacional del insecto (Aldemir \& Boşgelmez 2006, Gama et al. 2007).

Con relación a la población del insecto trasmisor de la enfermedad y los factores del clima en Chaco Argentina, se encontró una abundancia máxima (70\%) de huevecillos de $A$. aegypti en Noviembre y Diciembre del 2002, a una temperatura de $26.4^{\circ} \mathrm{C}$ y una precipitación de $270.3 \mathrm{~mm}$, en donde la precipitación es el factor que determina la presencia del insecto y el riesgo epidemiológico de la enfermedad con un valor de correlación de $r=0.57 \quad(\mathrm{p}<0.05)$, la cual coincide con la aparición de personas infectadas (Stein et al. 2005). En Barbados Argentina, se encontró este mismo comportamiento con una correlación máxima de $\mathrm{r}=0.25$ después de siete semanas (Depradine \& Lovell 2004). Así mismo, en Indonesia de 1992 a 2001 se determinó que la temperatura, precipitación, humedad y el índice de oscilación meridional tuvieron una $\mathrm{r}=0.20$ a $0.43(\mathrm{p}<0.05)$ después de seis meses de presentarse la máxima abundancia de mosquitos; la precipitación determinó la distribución geográfica y el patrón temporal de la incidencia, mientras que la temperatura influyó sólo en la intensidad del brote (Arcari et al. 2007). En San Juan, Puerto Rico la variación estacional promedio de los casos de DC/DH estuvieron relacionados con la estación de lluvias $\left(r^{2}=0.88\right)$ (Schreiber 2001).

En México, Hurtado-Díaz et al. (2007) estudiaron durante 1995 a 2003 el impacto de las variables del tiempo y de los indicadores climáticos asociados a la incidencia semanal del dengue en dos municipios de Veracruz, en el cual se encontró que el incremento de la temperatura superficial del mar (TSM) y la precipitación mínima semanal, fueron los factores que más se relacionaron con los casos de dengue, y que el tiempo de retraso entre las variables ambientales y la incidencia del virus registró un aumento de un grado centígrado en la TSM y ésta con el número de casos; en el municipio de San Andrés Tuxtla fue en 16 semanas $(46 \%, \mathrm{p}=0.001)$, y en el municipio de Veracruz fue de 20 semanas $(42 \%, \mathrm{p}=0.002)$, esto sugiere que se debe considerar a esta variable como medida predictiva de los casos de la enfermedad. Por otro lado, Peterson et al. (2005) realizaron estudios sobre la amplitud del nicho ecológico del insecto, y se generaron modelos de predicción con base en la temperatura, distribución espacial del vector y los casos de dengue.

El presente trabajo tuvo por objetivo: estudiar la abundancia y distribución de $A$. aegypti, así como conocer la dispersión y riesgo de la enfermedad en la ciudad de Guasave Sinaloa, México; para contar con información local sobre la población del insecto vector del dengue, que permita implementar mejores medidas de control.

\section{MATERIALES Y MÉTODOS}

Área de estudio: La ciudad de Guasave se ubica a los $25^{\circ} 10^{\prime} 03^{\prime \prime}$ - 25'46'19" N-108 $10^{\prime} 00^{\prime \prime}$ - 10906'50" W, en el Estado de Sinaloa. Cuenta con una superficie de $720 \mathrm{~km}^{2}$ y una población de 66793 habitantes (Fig. 1). Su clima es BW(h') seco muy cálido y cálido, el municipio recibe una precipitación pluvial media, máxima y mínima de 392.8, 760.0 y $231.1 \mathrm{~mm}$. La vegetación es un bosque espinoso que ha sido reemplazado por tierras de cultivo y un bosque de galería a lo largo del Río Sinaloa (INEGI 2005).

\section{Determinación del tamaño de muestra:} Para conocer el tamaño mínimo de muestra representativa de la población de habitantes en la ciudad se utilizó la fórmula de muestreo aleatorio simple (MAS) (Chapilliquén 2002). Por lo tanto, se incluyeron los seis sectores en los que se encuentra dividida la ciudad 


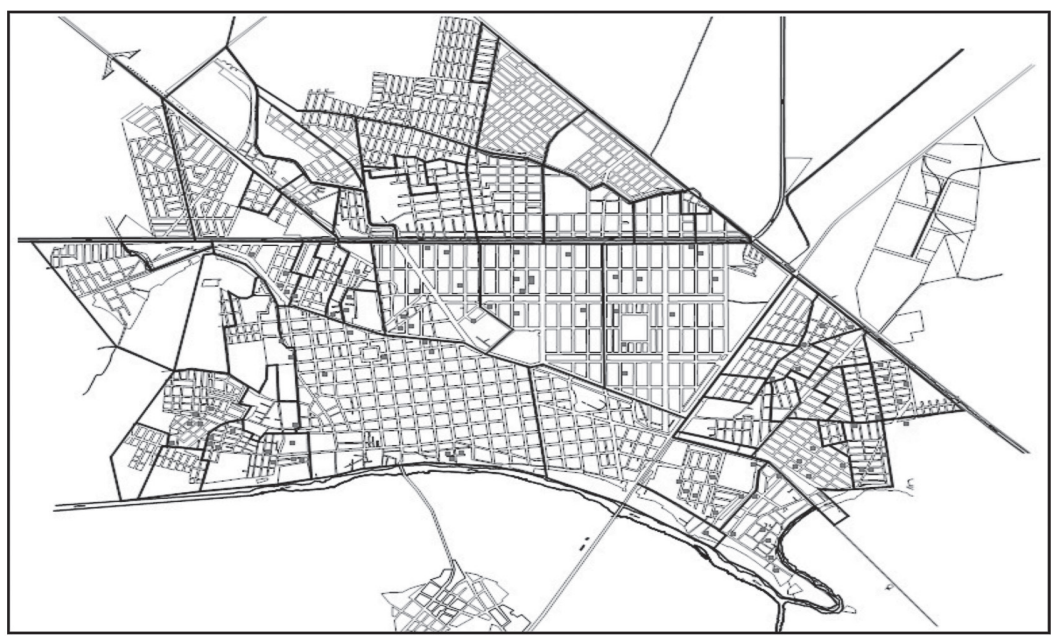

Fig. 1. Ciudad de Guasave y sitios con trampas por sector para recolecta de A. aegypti.

Fig. 1. Guasave city and sites with traps per sector to collect A. aegypti.

(Cuadro 1). El tamaño de la muestra (n) se estimó de la siguiente manera:

Fórmulas:

Ec. $1 \quad n=\frac{n^{\prime}}{\left(\frac{1+n^{\prime}}{N}\right)}$

Ec. 2

$$
n^{\prime}=\frac{S^{2}}{\mathrm{~V}^{2}}
$$

Donde:

$n$ : Tamaño de muestra

$S^{2}$ : Varianza de la muestra en términos de probabilidad $p(1-p)$

$s e$ : Error estándar

$\mathrm{V}^{2}$ : Varianza poblacional $\rightarrow(s e)^{2}$

$s e=0,05$

$\mathrm{N}=66$

Sustitución de Ec. 2

$$
n^{\prime}=\frac{0.05}{0.0025}=20
$$

Sustitución de Ec. 1

$$
n=\frac{20}{\left(\frac{1+20}{66}\right)}=15.4
$$

Debido al redondeo del número mayor se consideraron 16 colonias. Por su extensión la colonia Centro se dividió en los sectores 3 y 6 .

De esta manera se seleccionaron en cada sector las colonias con el mayor número de casas por manzana (Cuadro 1). En total se colocaron 96 trampas distribuidas en los seis sectores, 16 trampas por sector, las coordenadas geográficas de los sitios en los que se colocaron las trampas se determinaron con la utilización de un GPS Garmin 76 (Fig. 1).

Abundancia y distribución de A. aegypti: Para conocer la abundancia y distribución poblacional del mosquito se usó la técnica de trampas pegajosas, las cuales permitieron la recolecta y cuenta directa del número de hembras que se atraparon (Ritchie et al. 2003).

Cálculo de los índices IDA e ITP: El cálculo del índice de densidad de adultos 
CUADRO 1

Sectores y número de habitantes por colonia en Guasave

TABLE 1

Sectors and population per colony in Guasave

\begin{tabular}{clrr} 
Sectores & \multicolumn{1}{c}{ Colonias } & Casas & Habitantes \\
1 & La Piedrera, Revolución Mexicana, Independencia & 1102 & 4022 \\
2 & 2 de Octubre, Ayuntamiento 92 La Florida, Sauces & 3080 & 11042 \\
3 & Centro & 2861 & 8540 \\
4 & Del Bosque, Ejidal & 6105 & 16680 \\
5 & 18 de Marzo, Las Huertas, Ángel Flores & 3801 & 11636 \\
6 & Centro, Jardines del Valle, Tierra y Libertad & 4161 & 11198 \\
\hline
\end{tabular}

Fuente: Depto. de prevención y control de enfermedades transmitidas por vectores y zoonosis. Guasave Sinaloa, 2009.

(IDA) se realizó con el conteo del número de machos y hembras semana-mes por sector, este índice permitió conocer además la densidad poblacional mensual del vector (Aldemir \& Boşgelmez 2006, Gama et al. 2007). El índice de trampas positivas (ITP) por sector se determinó con base al número de hembras, lo que también permitió conocer la distribución la especie de abril de 2008 a marzo de 2009 en la ciudad de Guasave.

La trampa utilizada fue un diseño modificado de Facchinelli et al. (2007) tipo cubeta cubierta con una tapadera de $12 \mathrm{~cm}$, al recipiente se le agregaron $500 \mathrm{~mL}$ de agua de la llave como atrayente efectivo para las hembras; contenía además una tarjeta plástica de $10 \mathrm{~cm}$ de ancho a la cual se le aplicó un pegamento comercial formulado para fijar insectos PEGAFIN $50^{\circledR}$ (Maciel-de-Freitas et al. 2008). Cada semana se revisaron las trampas in situ y se consideró como trampa positiva a la que presentó por lo menos una hembra (Carbajo et al. 2006). Las trampas se colocaron en abril del 2008 con la colaboración del personal del departamento de vectores y zoonosis número dos de la Secretaría de Salud y Asistencia (SSA); éstas se colocaron en patios particulares, debido a que las hembras de A. aegypti buscan un sitio de ovoposición alrededor del domicilio (Maciel-de-Freitas et al. 2006). Las condiciones para colocar las trampas fueron homogéneas, se tomaron medidas preventivas para que los lugares seleccionados fueran áreas protegidas del viento y de la luz del sol. Cada mes se reemplazó la estructura interna con aplicación del nuevo pegamento, y se verificó su funcionamiento. Las trampas que fueron encontradas sin agua o destruidas se remplazaron y descartaron para el análisis estadístico (Facchinelli et al. 2007).

Correlación de la población de mosquito con la precipitación y temperatura: Así bien, se calculó el coeficiente de correlación de Pearson entre el número de hembras capturadas por mes y el valor acumulado de la precipitación y el promedio mensual de temperatura (media, máxima y mínima) proporcionados por la Comisión Nacional del Agua (CNA). Para obtener el coeficiente de correlación entre la densidad poblacional de A. aegypti y estos factores se utilizó el programa Excel (Arcari et al. 2007). El tiempo de retraso se consideró como el período extrínseco de incubación del virus, o tiempo que transcurre entre su entrada en el vector y el momento en el que puede ser transmitido, además de considerar la esperanza de vida del mosquito (Carbajo et al. 2001).

Correlación de la población de mosquitos con los casos de dengue: Los datos del número de trampas positivas y número de 
hembras fue relacionado con la información epidemiológica de casos de dengue en la ciudad, para determinar el grado de las asociaciones entre las variables climáticas y los casos de dengue clásico (DC) y dengue hemorrágico $(\mathrm{DH})$, con cero a seis meses de retraso (Depradine \& Lovell 2004, Rosa-Freitas et al. 2006). Los datos de dengue considerados fueron sólo los casos confirmados por la Secretaría de Salud y Asistencia (Dibo et al. 2008).

Identificación de las zonas de riesgo epidemiológico: Los valores totales del IDA e ITP por sector sirvieron para elaborar los mapas mensuales de dispersión de la enfermedad de abril del 2008 a marzo del 2009, la ubicación de los sitios donde se colocaron las trampas por sector se determinó usando el Sistema de Información Geográfica (SIG, que incorpora datos al programa Arc View SIG); se consideró como sector positivo $(\mathrm{P})$ cuando el valor del ITP fue $>0$ y negativo $(\mathrm{N})$ cuando fue $=0$ (Carbajo et al. 2004). De esta manera se generaron los polígonos de riesgo epidemiológico para los seis sectores de la ciudad, no obstante en este estudio se presenta sólo los mapas para los meses con el valor máximo y mínimo de IDA, así como un mapa de ITP donde se muestra el número de casos por sector a lo largo de los 12 meses de estudio.

\section{RESULTADOS}

Abundancia y distribución de A. aegypti: Durante el periodo de tiempo del estudio se encontraron 523 mosquitos de A. aegypti, de los cuales 472 (90\%) fueron hembras y $51(10 \%)$ machos. La mayor abundancia de hembras fue en julio y agosto de 2008 (47\%), la cual coincide con la mayor temperatura media mensual de 30.6 y $29.9^{\circ} \mathrm{C}$; en agosto y septiembre se registró la mayor precipitación acumulada (100 y 164mm) (Figs. 2 y 3 ).

Índice de adultos (IDA) e índice de trampas positivas (ITP): En julio y agosto se presentaron los valores más altos del IDA en los sectores dos (2.44), cuatro (3.35) y seis (2.290), mientras que en el sector tres, donde se ubica la colonia centro, de mejor condición socioeconómica, se registró el menor índice (0.0) (Cuadro 2). En agosto y septiembre los sectores $2(56.25), 4(58.82)$ y 6(61.54) presentaron los valores más altos de ITP (Cuadro 3 ), lo que además indica el periodo de la mayor

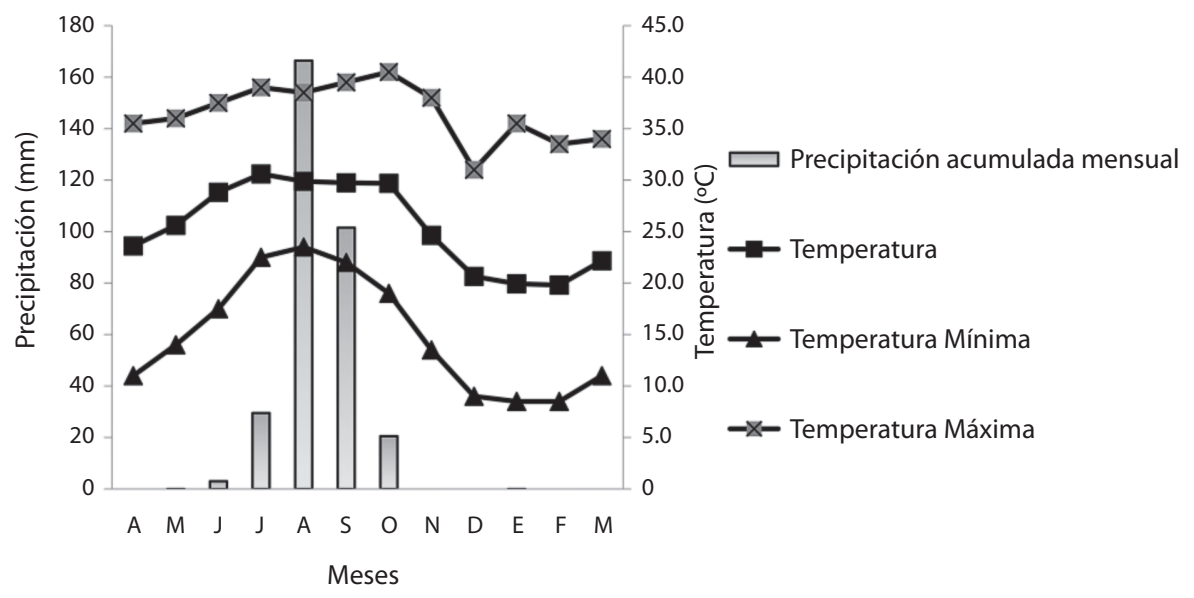

Fig. 2. Precipitación acumulada y temperaturas mensuales en Guasave, Sin.

Fig. 2. Precipitation and temperatures per month in Guasave, Sin.

Fuente: Comisión Nacional del Agua (CNA). 


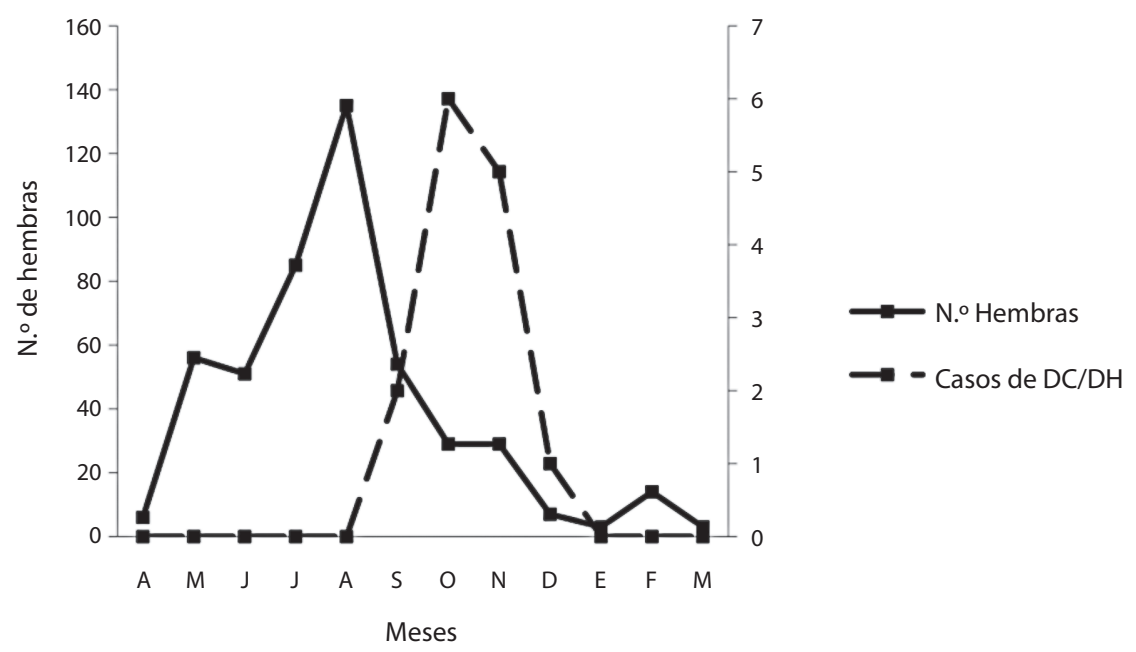

Fig. 3. Abundancia de hembras de A. aegypti y casos de DC/DH en Guasave, Sin.

Fig. 3. Abundance of females of A. aegypti and cases of DC/DH in Guasave, Sin.

CUADRO 2

Índice mensual de adultos (IDA) de A. aegypti por sector

TABLE 2

Monthly adult index (ADI) of A. aegypti per sector

\begin{tabular}{cccccccccccccc} 
Sector & A & M & J & J & A & S & O & N & D & E & F & M \\
1 & 0.00 & 0.67 & 0.00 & 0.92 & 1.00 & 0.42 & 0.20 & 0.30 & 0.18 & 0.00 & 0.27 & 0.11 \\
2 & 0.00 & 0.10 & 0.20 & 0.94 & 2.44 & 1.06 & 0.56 & 0.25 & 0.06 & 0.06 & 0.31 & 0.07 \\
3 & 0.00 & 0.33 & 0.20 & 0.00 & 0.33 & 0.25 & 0.00 & 0.50 & 0.67 & 0.00 & 0.00 & 0.00 \\
4 & 0.06 & 0.22 & 1.85 & 0.77 & 3.35 & 0.78 & 0.65 & 0.12 & 0.07 & 0.00 & 0.29 & 0.00 \\
5 & 0.00 & 0.44 & 0.31 & 1.31 & 0.81 & 0.44 & 0.73 & 0.82 & 0.00 & 0.00 & 0.00 & 0.00 \\
6 & 0.26 & 2.00 & 0.86 & 2.29 & 1.31 & 0.71 & 0.47 & 0.33 & 0.17 & 0.15 & 0.17 & 0.08 \\
\hline
\end{tabular}

CUADRO 3

Índice mensual de trampas positivas (ITP) de A. aegypti por sector

TABLE 3

Month index positive traps (PTI) of A. aegypti per sector

\begin{tabular}{cccccccccccccc} 
Sector & A & M & J & J & A & S & O & N & D & E & F & M \\
1 & 0.00 & 33.33 & 0.00 & 33.33 & 37.50 & 25.00 & 2.00 & 2.00 & 9.09 & 0.00 & 8.18 & 0.11 \\
2 & 0.00 & 10.00 & 2000 & 31.25 & 56.25 & 50.00 & 31.25 & 12.50 & 5.88 & 5.88 & 0.06 & 0.07 \\
3 & 0.00 & 33.00 & 20.00 & 0.00 & 33.33 & 25.00 & 0.00 & 50.00 & 66.67 & 0.00 & 0.00 & 0.00 \\
4 & 5.56 & 16.67 & 6.67 & 38.46 & 58.82 & 50.00 & 28.57 & 11.76 & 7.14 & 0.00 & 0.29 & 0.00 \\
5 & 0.00 & 12.50 & 13.33 & 35.71 & 37.50 & 25.00 & 36.36 & 23.53 & 0.00 & 0.00 & 0.00 & 0.00 \\
6 & 10.53 & 50.00 & 7.69 & 42.86 & 61.54 & 57.14 & 33.33 & 16.67 & 16.67 & 33.33 & 0.17 & 0.25 \\
\hline
\end{tabular}


distribución del vector, precisamente cuando las lluvias fueron más abundantes, esto originó una abundancia de criaderos para la reproducción del insecto. En diciembre, en el sector tres se registró el valor más alto de ITP con un 66.67; este valor fue alto pero por si solo no indica la distribución del insecto en el área de estudio.

Correlación entre la abundancia poblacional de A. aegypti y los casos de DC y DH: En el periodo de estudio se reportaron 14 casos de dengue en Guasave, ocho casos fueron de DC y seis de DH, el máximo número de hembras se registró en agosto (140) y el máximo número de casos de $\mathrm{DC} / \mathrm{DH}$ en octubre (seis) por lo que hubo un desfase de dos meses entre ellos (Fig. 3). El análisis de correlación de Pearson $(\mathrm{p}<0.05)$ entre la abundancia poblacional de hembras (AP) y los casos de DC y DH, en el cual se consideraron cero a seis meses de retraso, indicó que las correlaciones de mayor asociación fueron principalmente a dos y tres meses de retraso $(0.88,0.90)$, aunque para la abundancia poblacional AP con el $\mathrm{DH}$, se obtuvo una correlación considerable a los cuatro meses de retraso 0.58 (Cuadro 4).

Correlación entre la abundancia poblacional de A. aegypti y los casos de DC/DH con la precipitación y la temperatura: En el Cuadro 4 se observa el grado de las asociaciones entre la precipitación y temperatura con los casos de DC y DH de cero a seis meses de retraso en la región de estudio. Los resultados indicaron que las mayores correlaciones fueron desde cero a cuatro meses de retraso, pero la mayor correlación se obtuvo entre la abundancia del mosquito con la precipitación y temperatura mínima a cero meses de retraso $(0.80,0.84)$. La mayor correlación entre la abundancia poblacional y los casos de DC/DH se obtuvo a los tres meses de retraso, mientras que con la precipitación (0.94) fue a dos meses de retraso y con la temperatura mínima (0.84) a cero meses.

Mapas de dispersión y riesgo epidemiológico: Con los índices de IDA e ITP y los casos de dengue se elaboraron mapas

CUADRO 4

Correlación entre la poblacional de A. aegypti (AP) con los casos de DC/DH, la abundancia de hembras, precipitación y temperatura

TABLE 4

Correlation between A. aegypti population (AP) with the CD/HD cases, female abundance, precipitation and temperature

\begin{tabular}{|c|c|c|c|c|c|}
\hline & \multirow{2}{*}{ Número de hembras } & \multirow{2}{*}{ Precipitación (mm) } & \multicolumn{2}{|c|}{ Temperatura $\left({ }^{\circ} \mathrm{C}\right)$} & \multirow{2}{*}{ Promedio } \\
\hline & & & Mínima & Máxima & \\
\hline \multirow[t]{2}{*}{ AP } & & $0.80(0)$ & $0.84(0)$ & $0.57(0)$ & $0.76(0)$ \\
\hline & & $0.98(2)$ & $0.79(2)$ & $0.66(1)$ & $0.64(2)$ \\
\hline \multirow[t]{2}{*}{$\mathrm{DC} / \mathrm{DH}$} & $0.78(2)$ & & $0.75(3)$ & $0.54(2)$ & $0.66(3)$ \\
\hline & $0.85(3)$ & $0.75(1)$ & $0.65(1)$ & $0.61(0)$ & $0.58(1)$ \\
\hline \multirow[t]{2}{*}{ DC } & $0.88(2)$ & $0.94(2)$ & $0.77(2)$ & $0.59(1)$ & $0.61(2)$ \\
\hline & $0.69(3)$ & & $0.64(3)$ & & $0.60(3)$ \\
\hline \multirow[t]{3}{*}{$\mathrm{DH}$} & $0.51(2)$ & $0.96(2)$ & $0.67(2)$ & $0.63(1)$ & $0.57(2)$ \\
\hline & $0.90(3)$ & & $0.76(3)$ & $0.54(2)$ & $0.61(3)$ \\
\hline & $0.58(4)$ & & $0.58(4)$ & & $0.58(4)$ \\
\hline
\end{tabular}

(1-4)=Meses de retraso.

$\mathrm{AP}=$ abundancia de $A$. aegypti, $\mathrm{DC}=$ dengue clásico, $\mathrm{DH}=$ dengue hemorrágico. 
mensuales de dispersión de la enfermedad, únicamente con el objeto de mostrar la magnitud del riesgo de la enfermedad en el tiempo y en el espacio. No obstante, en este trabajo sólo se presentan los mapas de IDA de agosto, donde se registraron los valores más altos (2.44-3.35), y en marzo con los valores más bajos (0.007$0.08)$, también se apreció que los sectores más vulnerables en agosto fueron los sectores dos, cuatro y seis (Fig. 4A), los cuales corresponden a lugares donde habitan familias de bajos recursos, no obstante, en marzo los de menor vulnerabilidad fueron el tres, cuatro y cinco que corresponde a colonias con mayor nivel socioeconómico (Fig. 4B). Los valores positivos de ITP (expresado en porcentaje) indicaron que los meses de mayor riesgo epidemiológico fueron julio (14\%), agosto (22\%) y septiembre (17\%). En la Fig. 5 se muestra que las zonas de riesgo para agosto con altos valores positivos de ITP fueron los sectores dos, cuatro y seis.

\section{DISCUSIÓN}

La mayor abundancia del mosquito fue en julio, agosto y septiembre de 2008 (47\%), lo cual coincide con la mayor temperatura media mensual de $30.6^{\circ} \mathrm{C}$ en la época con mayor precipitación acumulada (100-164mm), dos meses después, se presentó el mayor número de casos de la enfermedad. Estos resultados concuerdan con los obtenidos por Peterson et al. (2005), quienes encontraron que cuando la distribución del insecto es amplia existe mayor número de casos de dengue.

La mayor actividad de apareamiento y ovipostura de A. aegypti se observó a temperaturas de $29^{\circ} \mathrm{C} \mathrm{y} 30^{\circ} \mathrm{C}$, lo que sugiere que esta es óptima para la actividad reproductiva del insecto. En concordancia con esto, Micieli et al. (2006) encontraron que temperaturas bajas de $13^{\circ} \mathrm{C}$ a $15^{\circ} \mathrm{C}$ no tuvieron correlación significativa con el vector.
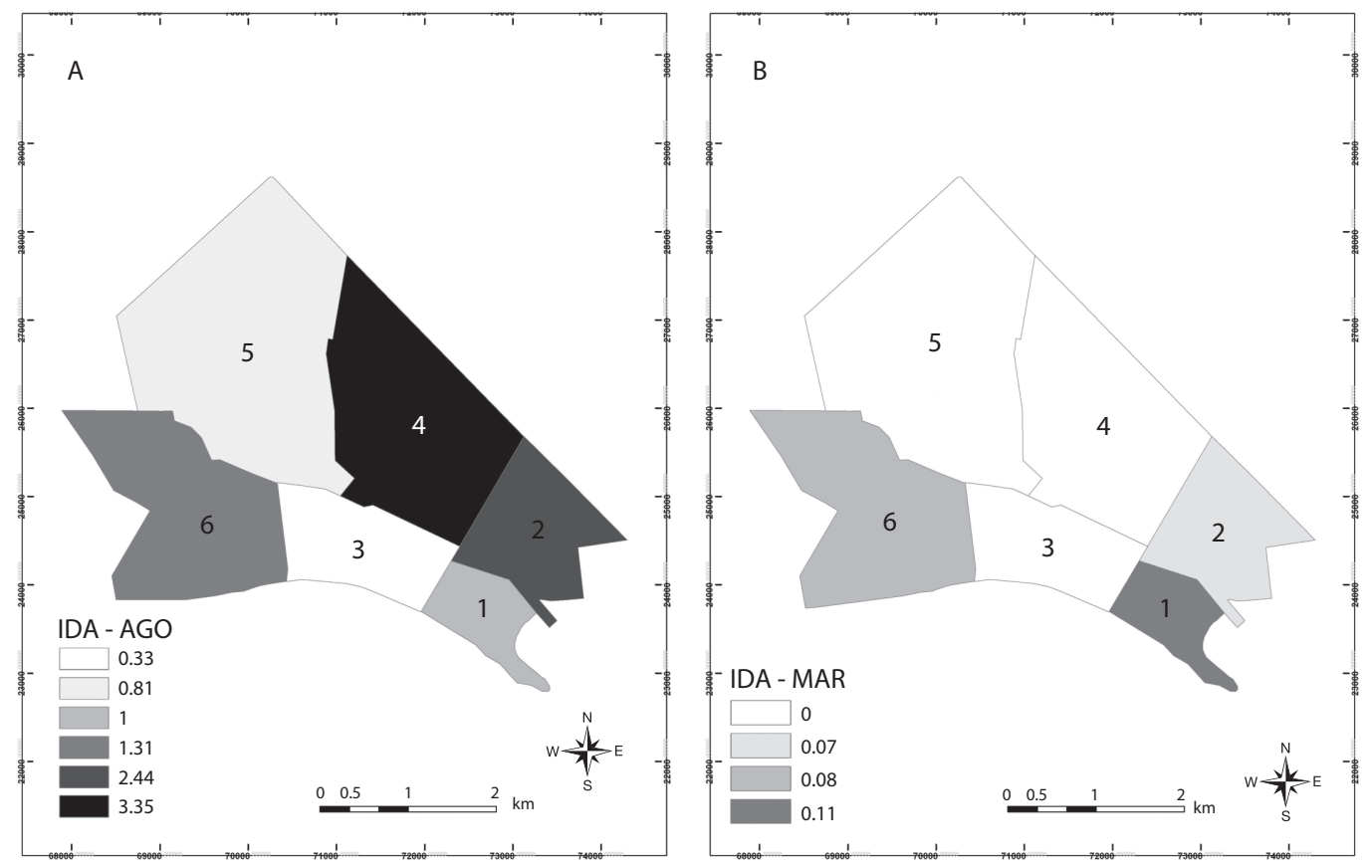

Fig. 4. (A) Valores máximos de IDA y riesgo de dispersión de dengue por sector en Guasave, Sin. (B) Valores mínimos de IDA y dispersión de dengue en Guasave, Sinaloa.

Fig. 4. (A) Maximum ADI values, and risk dispersion of dengue per sector in Guasave, Sin. (B) Minimum ADI values, and risk dispersion of dengue per sector in Guasave, Sinaloa. 


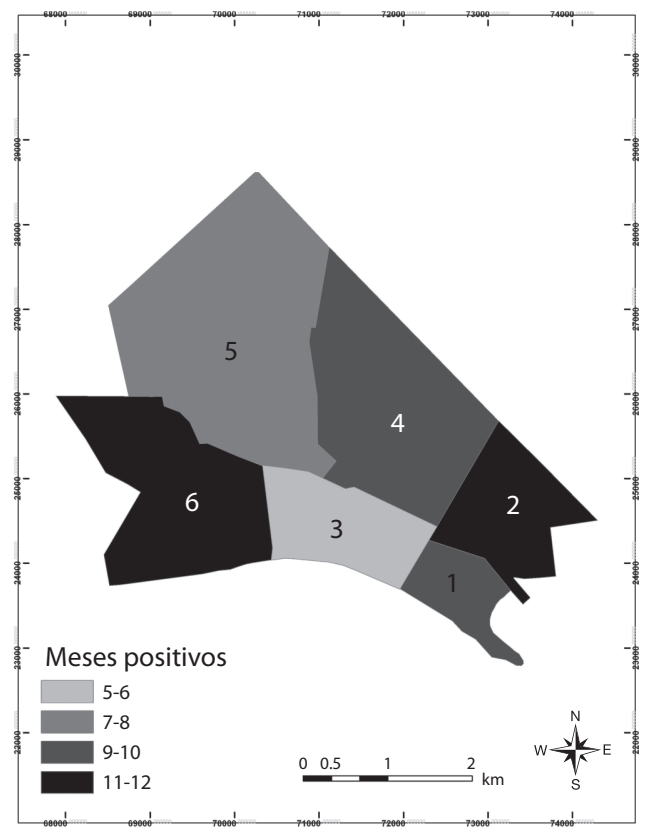

Fig. 5. Meses con trampas positivas (ITP) por sector en Guasave, Sin.

Fig. 5. Months with positive traps index (PTI) per sector in Guasave, Sin.

La mayor correlación de la densidad de hembras se obtuvo con la precipitación acumulada $(r=0.80)$ a cero meses de retraso, lo que demostró que los periodos de lluvia fueron los de mayor riesgo epidemiológico para la transmisión del dengue en el lugar de estudio, donde además se detectó un mayor número de trampas positivas y se observó también un intensa actividad de hembras que se disponían a ovipositar. Estos resultados coincidieron con los de Stein et al. (2005), quienes obtuvieron mayor número de huevecillos en la temporada de mayor precipitación acumulada mensual.

Schreiber (2001), encontró que la variación estacional de los casos de DC/DH está muy relacionada con la precipitación, con un tiempo de retraso de un mes, en tanto que en nuestros resultados la mayor relación se obtuvo a dos meses de retraso $(\mathrm{r}=0.98)$, por lo que esta variable sirvió como medida de predicción rápida de la enfermedad.
Rosa-Freitas et al. (2006) obtuvo correlaciones significativas $(\mathrm{r}=0.072-0.571)$ entre el número de casos de dengue y el clima, las cuales fueron de bajas a moderadas y dependieron de los siguientes factores: período del año, la variable climática, tiempo de retraso y tiempo en que aparece la enfermedad. En nuestro caso se encontraron correlaciones mayores ( $r=0.54-0.98)$ debido a que en dos meses aparecieron los síntomas de la enfermedad. Por esta razón, se considera que las correlaciones máximas entre casos de DH obtenidas con tres y cuatro meses de retraso son una medida tardía en la predicción de casos.

En resumen, podemos afirmar que la precipitación acumulada y la temperatura mínima sirven como medida predictiva de la población del vector y como medida de la dispersión del dengue a dos meses de retraso.

Los casos de dengue y el cálculo mensual de los índices IDA e ITP en los seis sectores, permitieron conocer la dispersión y riesgo epidemiológico de la enfermedad en la ciudad. En trabajos subsecuentes pretendemos evaluar la seropositividad del vector para correlacionarlo con la presencia de casos clínicos, a través de un análisis estadístico multivariado.

\section{AGRADECIMIENTOS}

Este trabajo se realizó con el apoyo financiero del Consejo Estatal de Ciencia y Tecnología del estado de Sinaloa (CECyT). Proyecto: "Biología, ecología y control biológico del mosquito Aedes aegypti (L) en Guasave, Sinaloa".

\section{RESUMEN}

El dengue es una enfermedad viral en humanos transmitida por el mosquito A. aegypti (L), principal vector en América. En el 2006 en Sinaloa México se presentaron 477 casos de dengue hemorrágico y 1510 de dengue clásico, el estado presentó el mayor número de casos de dengue hemorrágico del país, debido a esto se realizó un estudio sobre la abundancia y distribución del mosquito de abril 2008 a agosto 2009 en Guasave Sinaloa, México. La Ciudad se dividió en seis sectores, en la cual se consideraron 16 colonias con el mayor número de casas por sector; 
se colocaron 96 trampas para monitorear la densidad de población del insecto y el número de hembras por trampa, esto datos sirvieron para calcular los índices de densidad de adultos (IDA) e índice de trampas positivas (ITP) por sector, los cuales junto con los casos de dengue permitieron elaboraron mapas de dispersión de la enfermedad. En julio y agosto se registró la abundancia más alta de hembras (137 y 139). En agosto y septiembre los sectores dos, cuatro y seis presentaron valores de IDA de 2.44, 3.35 y 2.29, por lo que fueron de un alto riesgo epidemiológico, mientras que en estos mismos sectores se encontraron los valores más altos de ITP $(56.25,58.82$ y 61.54$)$. Los valores de mayor asociación de las correlaciones entre la abundancia poblacional de hembras se obtuvo con la precipitación $(\mathrm{r}=0.80)$ y con la temperatura promedio $(\mathrm{r}=0.76)$, a cero meses de retraso. La correlación entre el DC/DH con la precipitación fue más alta ( $\mathrm{r}=0.98)$ que con la temperatura mínima $(r=0.79)$; ambas con dos meses de retraso. En este estudio se demostró que los mapas mensuales de dispersión epidemiológica sirven como una medida del riesgo de la enfermedad en Guasave, Sinaloa.

Palabras clave: mosquito, dispersión, dengue clásico, dengue hemorrágico, Guasave Sinaloa México.

\section{REFERENCIAS}

Aldemir, A. \& A. Boşgelmez. 2006. Population Dynamics of Adults and Immature Stages of 11Mosquitoes (Diptera:Culicidae) in Gölbaşi District, Ankara. Turk. J. Zool. 30: 9-17.

Arcari, P., N. Tapper \& S. Pfueller. 2007. Regional variability in relationships between climate and dengue/ DHF in Indonesia. J. Trop. Geo. 28: 251-272.

Carbajo, A.E., N.J. Schweigmann, S.I. Curto, A. de Garín \& R. Bejarán. 2001. Dengue transmission risk maps of Argentina. Trop. Med. Int. Health 6: 170-183.

Carbajo, A.E., S.M. Gomez, S.I. Curto \& N.J. Schweigmann. 2004. Variación espacio-temporal del riesgo de transmisión del dengue en la Ciudad de Buenos Aires. Med. Int. Health 64: 231-234.

Carbajo, A.E., S.I. Curto \& N.J. Schweigmann. 2006. Spatial distribution pattern of oviposition in the mosquito Aedes aegypti in relation to urbanization in Buenos Aires: southern fringe bionomics of an introduced vector. Med. Vet. Entomol. 20: 209-218.

Chapilliquén, F. 2002. Comparativo de metodologías para el levantamiento del Índice Aédico en la localidad Pampa Huasa Huasi. Direccion de Salud Junín. Rev. Peru. Epidemiol. 10: 1-7.
Depradine, C. \& E. Lovell. 2004. Climatological variables and the incidence of Dengue fever in Barbados. Int. J. Environ. Health Res. 14: 429-441.

Dibo, M.R., A.P. Chierotti, M.S. Ferrari, A.L. Mendonça \& F.C. Neto. 2008. Study of the relationship between Aedes (Stegomyia) aegypti egg and adult densities, dengue fever and climate in Mirassol, state of São Paulo, Brazil. Mem. Inst. Osw. Cruz 103: 554-560.

Facchinelli, L., L. Valerio, M. Pombi, P. Reiter, C. Costantini \& A. Dellatorre. 2007. Development of a novel sticky trap for containerbreeding mosquitoes and evaluation of its sampling properties to monitor urban populations of Aedes albopictus. Med. Vet. Entomol. 21: 183-195.

Focks, D.A. 2003. A review of entomological sampling methods and indicators for dengue vectors. World Health Organization, Ginebra, Suiza.

Gama, R.A., E.M. Silva, I.M. Silva, M.C. Resende \& A.E. Eiras. 2007. Evaluation of the Sticky MosquiTRAP $^{\mathrm{TM}}$ for Detecting Aedes (Stegomyia) aegypti (L.) (Diptera: Culicidae) during the Dry Season in Belo Horizonte, Minas Gerais, Brazil. Neotrop. Entomol. 36: 294-302.

Hurtado-Díaz, M., H. Riojas-Rodríguez, S.J. Rothenberg, H. Gomez-Dantés \& E. Cifuentes. 2007. Short communication: Impact of climate variability on the incidence of dengue in Mexico. Trop. Med. Int. Health 12: 1327-1337.

INEGI. 2005. Aspectos geograficos de Guasave. Instituto Nacional de Estadísticas y Geografía, Aguascalientes, México.

Maciel-de-Freitas, R., A. Eduardo-Eiras \& R. Lourençode-Oliveira. 2006. Field evaluation of effectiveness of the BG-Sentinel, a new trap for capturing adult Aedes aegypti (Diptera: Culicidae). Mem. Inst. Osw. Cruz 101: 321-325.

Maciel-de-Freitas, R., R. Costa-Peres, F. Alves \& M. Blanco-Brandolini. 2008. Mosquito traps designed to capture Aedes aegypti (Diptera: Culicidae) females: preliminary comparison of Adultrap, MosquiTRAP and backpack aspirator efficiency in a dengue-endemic area of Brazil. Mem. Inst. Osw. Cruz 103: 602-605.

Micieli, M.V., J.J. García \& G.A. Martí. 2006. Dinámica poblacional de los estadios inmaduros del vector del dengue Aedes aegypti (Diptera: Culicidae): un estudio longitudinal (1996-2000). Rev. Biol. Trop. 54: 979-983. 
Peterson, T.A., C. Martínez-Campos, Y. Nakazawa \& E. Martínez-Meyer. 2005. Time-specific ecological niche modeling predicts spatial dynamics of vector insects and human dengue cases. Trop. Med. Hyg. 99: 647-655.

Ritchie, S.A., C.E. Webb \& R.C. Russell. 2003. An adulticidal sticky ovitrap for sampling containerbreeding mosquitoes. J. Am. Mosq. Control. Assoc. 19: $235-242$.

Rosa-Freitas, M.G., K.V. Schreiber, P. Tsouris, E.T. de Souza-Weimann \& J.F. Luitgards-Moura. 2006. Associations between dengue and combinations of weather factors in a city in the Brazilian Amazon. Rev. Panam. Salud Publica 20: 256-267.
Schreiber, K.V. 2001. An investigation of relationships between climate and dengue using a water budgeting technique. Int. J. Biom. 45: 81-89.

SSA. Secretaría de Salud. 2007. Manual para la Vigilancia, Diagnóstico, Prevención y Control del Dengue. Secretaría de Salud, México, D.F., México.

Stein, M., G.I. Oria \& J.A. Willener. 2005. Fluctuación estacional de Aedes aegypti en Chaco, Argentina. Rev. Saú. Públ. 39: 559-564.

WHO. World Health Organization. 1995. Guidelines for dengue surveillance and mosquito control. World Health Organization, Regional Office for the Western Pacific, Manila, Filipinas. 
